\title{
Yod
}

Revue des études hébraïques et juives

$14 \mid 2009$

La littérature israélienne, miroir d'une société multiple

\section{Stratégies littéraires dans la littérature féminine israélienne}

Le cas d'Orly Castel-Bloom

Literary Strategies in Women's Israeli Literature: Orly Castel-Bloom

אסטרטגיות ספרותית בכתיבת נשים ישראלית

\section{Elisa Carandina}

\section{(2) OpenEdition}

\section{Journals}

Édition électronique

URL : https://journals.openedition.org/yod/393

DOI : 10.4000/yod.393

ISSN : 2261-0200

Éditeur

INALCO

Édition imprimée

Date de publication : 1 octobre 2009

Pagination : 233-246

ISBN : 978-2-85831-178-1

ISSN : 0338-9316

Référence électronique

Elisa Carandina, «Stratégies littéraires dans la littérature féminine israélienne », Yod [En ligne], 14 | 2009, mis en ligne le 01 novembre 2011, consulté le 08 juillet 2021. URL : http://

journals.openedition.org/yod/393; DOI : https://doi.org/10.4000/yod.393

Ce document a été généré automatiquement le 8 juillet 2021.

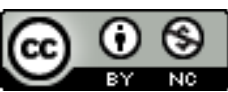

Yod est mis à disposition selon les termes de la Licence Creative Commons Attribution - Pas d'Utilisation Commerciale 4.0 International. 


\title{
Stratégies littéraires dans la littérature féminine israélienne
}

\author{
Le cas d'Orly Castel-Bloom \\ Literary Strategies in Women's Israeli Literature: Orly Castel-Bloom \\ אסטרטגיות ספרותית בכתיבת נשים ישראלית
}

\author{
Elisa Carandina
}

\section{Introduction}

1 Aujourd'hui, la littérature féminine israélienne constitue un domaine particulièrement complexe et hétérogène qui stimule la réflexion critique lorsque l'on s'efforce d'en comprendre les caractéristiques. L'une des constantes des diverses approches critiques semble être la volonté de retrouver dans cette littérature des éléments de contestation et de révolte à différents niveaux. Cette volonté précède bien souvent l'analyse et l'oriente. Le développement, ces dernières années en Israël, d'un féminisme influencé par les mouvements féministes dans le monde, allié à l'équation simpliste « littérature féminine " égale "littérature féministe militante ", ont conduit la critique à évaluer l'œuvre en fonction de sa dimension féministe. Le récent essor de la littérature féminine israélienne risque ainsi d'être uniquement considéré sous l'angle d'une révolte contre la tradition, ce qui aplanit les différentes positions et ne tient pour ainsi dire pas compte des éléments strictement littéraires.

2 Sur la base de ces préliminaires, l'un des moyens susceptibles d'évaluer cet élément de nouveauté dans la littérature féminine israélienne en lui rendant ses proportions pourrait être l'analyse des stratégies littéraires employées et, plus précisément, le rapport avec la tradition littéraire du mythe. En effet, certains traits de la littérature féminine en Israël peuvent être esquissés à partir d'un dialogue avec des archétypes littéraires afin de les localiser et de cerner de façon plus précise les stratégies littéraires de cette révolte que la littérature des femmes semble être destinée à incarner. 
3 En général, l'évocation de thèmes mythiques, à savoir la présence d'éléments évoquant un thème littéraire connu et reconnaissable, permet de construire un dialogue entre ce patrimoine et la nouvelle version du mythe. Le rapport avec le mythe peut donc être un moyen efficace de donner des coordonnées explicites permettant de positionner un texte dans la géographie littéraire grâce à l'appréciation des éléments de continuité et de discontinuité. À ce propos, on peut considérer l'élément de l'inventio comme une des clés interprétatives de ce parcours, en d'autres termes, la « découverte " d'éléments préexistants qui ne doivent donc pas être «inventés » ex nihilo, mais retrouvés et considérés dans le contexte approprié. Pour simplifier: lorsqu'une histoire est déjà connue, on peut mieux apprécier ses variations plus récentes.

En outre, la technique de la réécriture du mythe dans la littérature féminine est sans doute l'un des éléments que la critique considère comme fondamental, selon la définition qu'Alicia Ostriker donne du processus de revisionist mythmaking. On en trouve de nombreux exemples dans la littérature israélienne - il suffit dans ce contexte de mentionner l'usage du mythe de la « aqeda (le sacrifice d'Isaac) dans la poésie féminine ou la réécriture des autres patterns dans l'écriture des femmes religieuses. D'une part, cette stratégie évoque la volonté de s'approprier une tradition de laquelle les femmes se sont senties exclues jusqu'à présent, dans un désir de maitriser, voire de "voler » ce patrimoine culturel selon la célèbre expression utilisée par Hélène Cixous. D'autre part, cette appropriation pose la question de la validité de la tradition utilisée et de savoir dans quelle mesure elle découle d'une volonté de s'approprier pour modifier, de s'accaparer pour détruire. La stratégie de la réécriture du mythe se trouve ainsi au cœur d'une dynamique incarnant des forces opposées.

Cette perspective peut utilement être appliquée aux œuvres d'une femme écrivain considérée parmi les voix les plus provocatrices de la littérature israélienne contemporaine: Orly Castel-Bloom (née en 1960). Cet auteur a souvent bouleversé l'opinion publique et suscité un intérêt profond chez les critiques, de par sa volonté manifeste de s'approprier des thèmes-clés de la tradition culturelle israélienne et universelle dans le but de les réinterpréter, parfois jusqu'à les vider de leur signification. Ce jeu de répétitions et d'allusions à un système référentiel en train de s'effriter, mais assez puissant, car étant encore considéré comme «le » système de référence, construit un dialogue intéressant. Ce dialogue se base sur un non-dit constitué par un patrimoine culturel et social qui ne peut pas - ou pas encore - être ignoré et qui semble démontrer la puissance de la «carte qui désormais précède le territoire » selon la métaphore employée par Jean Baudrillard ${ }^{1}$.

\section{Le mythe}

6 Avant d'aborder l'analyse de certains aspects du mythe dans l'œuvre d'Orly CastelBloom, il convient de préciser dans quelle perspective on désire approfondir l'utilisation du mythe. Tout d'abord, il faut considérer le contexte postmoderne dans lequel le mythe est plongé. De ce point de vue, on ne pourrait pas l'aborder d'une façon innocente, à savoir en considérant le mythe comme un symbole susceptible de créer un nouveau système de signification. Le rapport signifiant-signifié est en effet impossible à reconstituer et tout ce qui reste est la structure du mythe. Souvent dans la littérature postmoderne on utilise, ou peut-être on utilisait, le mythe en ayant perdu la foi en son contenu, mais en croyant encore à sa possibilité d'exister en tant que structure 
rhétorique, en tant que moyen pour exprimer une distance, un éloignement de la tradition linguistique et culturelle. Le paradoxe, ainsi que la force de la présence du mythe dans cette littérature, est constitué par l'utilisation du mythe contre lui-même, du symbole en fonction antisymbolique. Ce court-circuit peut se vérifier sur le plan du contenu, et alors on peut parler de subversion du mythe, mais aussi en tant que réflexion sur le langage et sur sa nature métaphorique ${ }^{2}$.

Dans cette perspective, il est évident que le mythe devient un moyen privilégié pour aborder le thème du langage et de l'interprétation, vu que le langage du mythe est métaphorique et qu'il nécessite une interprétation ou, plus exactement, que la première signification du mythe est celle de la nature métaphorique du langage. Ainsi, analyser le rapport avec le mythe dans un roman postmoderne signifie analyser le rapport $\mathrm{du}$ texte, non seulement avec une tradition littéraire et un système de référence, mais aussi avec le langage en tant que moyen d'expression artistique. Les deux éléments peuvent être considérés comme les coordonnés géographiques de la position du texte dans la carte de la littérature pour ainsi dire, et ils sont donc particulièrement utiles dans notre perspective.

de cet article est en effet de mettre en relief quelques exemples de l'utilisation $\mathrm{du}$ mythe et de la métaphore dans l'œuvre d'Orly Castel-Bloom. Pour cette analyse, nous avons choisi le roman Dolly City $^{3}$ en ne considérant pas seulement la puissance du mythe dans le roman, mais aussi la fonction de manifeste littéraire pouvant être attribuée à ce roman.

En effet, les deux thèmes fondamentaux du roman, à savoir création littéraire et procréation, sont abordés par l'évocation des mythes qui peuvent aider à comprendre au mieux les différentes possibilités de la technique de la réécriture, considérée comme une des stratégies littéraires « classiques » de la littérature féminine ${ }^{4}$. De plus, le roman approfondit le thème du langage d'une façon qui éclaire le rapport de l'auteur avec la puissance des mots. Au-delà de la définition de réécriture grotesque du mythe donné par la critique, on essayera d'analyser dans le roman d'Orly Castel-Bloom quelques exemples susceptibles de nous aider à comprendre la technique de cette réécriture du mythe et le rapport de ce procédé avec le langage, d'une part, et avec le « revisionist mythmaking » dans la littérature féminine, d'autre part.

\section{Dolly City entre Déméter et Pygmalion}

Le thème de la perfection est notamment l'élément commun entre la recherche de Dolly en tant que mère/médecin et celle de Dolly en tant qu'incarnation de l'auteur. En effet, le thème de la procréation est strictement lié à celui de la création littéraire et la critique a déjà démontré la possibilité d'interpréter le personnage du Fils en tant que symbole de l'œuvre littéraire ${ }^{5}$. L'élan vers la perfection se manifeste d'un côté dans la revendication de Dolly de se charger jusqu'au bout de sa "responsabilité maternelle", d'être donc la meilleure des mères possible. Dans le contexte de Dolly City, ville violente et cruelle, ce rôle implique la capacité de faire survivre son propre fils, de le soustraire à une loi qui le voit déjà condamné, de le sauver des forces qui essaient de le kidnapper. La possibilité de créer un lien avec le mythe de Déméter et Perséphone au niveau de la structure narrative est donc possible sur la base de ces éléments, même si un parallèle ponctuel du point de vue des épisodes du roman n'est pas dans le but de cet article. L'évocation de cette force maternelle est celle de la seule force capable de sauver du fait 
qu'elle est la seule capable de donner la vie (mais aussi de la reprendre, en accord avec le mythe de la Déesse mère à la force tellurique) comme la critique l'a déjà souligné. Toutefois on pourrait parler plus correctement de la représentation d'une force maternelle considérée comme menaçante parce que sa volonté de protection n'est pas comprise. Donc, une Dolly qui évoque le personnage de Déméter qui dans l'Hymne homérique jette dans le feu le fils de Celeus pour le rendre immortel même si les hommes, « aveugles et insensés », l'empêcheront d'accomplir son projet en craignant à tort pour la vie de l'enfant.

11 La présence de cette force maternelle incomprise, mais qui à la fin réussira à parvenir à son but grâce à son obstination, est très intéressante si on considère son lien avec l'autre mythe de perfection évoqué dans le roman, à savoir celui de Pygmalion. J'ai déjà traité ailleurs du lien entre la recherche de perfection de Dolly et le monstre de Frankenstein ${ }^{6}$. Ici, je voudrais approfondir la volonté d'Orly Castel-Bloom de conférer une nuance maternelle au mythe de l'artiste et de la création incarné par le mythe de Pygmalion. On retrouve dans le roman Dolly City le passage du mythe classique de Pygmalion au mythe romantique de la création, phénomène qu'Anne Geisler Szmulewicz a très bien illustré pour ce qui concerne la littérature française. ${ }^{7}$

Une des possibles lectures du rapport entre Dolly et son Fils est la recherche de perfection artistique à partir d'un idéal, d'une idée abstraite que la mère-artiste cherche à incarner dans la réalité. Fils est le "petit projet » de Dolly qui craint que quelqu'un, sous le prétexte d'être son grand-père, puisse venir le lui prendre. Ce projet est formulé sur la base de règles bien précises, scientifiques pour ainsi dire, parce qu'il est aussi une expérience, une combinaison du mythe de la création artistique et de celui de la recherche scientifique dans sa dimension prométhéenne. Dolly-Pygmalion - mais aussi Prométhée dans son aspect romantique de révolte contre les dieux - crée le Fils en tant qu'œuvre d'art qui peut être exposée, comme elle le fera par la suite. Comme la Galathée de Pygmalion, le Fils est l'obsession de Dolly, elle modèle son corps comme s'il était fait de marbre et non de chair vivante, dans le but de réaliser avec cet enfant un mirage de perfection. À l'instar de Pygmalion, Dolly recherche la fusion entre le créateur et son œuvre, fusion qui se " concrétise » dans l'épisode du collage de l'enfant au dos de la mère. Fils est son œuvre animée, il est l'expression de la vérité intérieure du créateur dans sa nature de "reflet de plusieurs années de folie ${ }^{8}$, il est la folle tentative de rendre chair aux idéaux auxquels on ne croit plus et qui n'existent plus. L'art comme représentation incarnée par Galathée est devenu impossible dans cet univers, ce qui reste est la simulation, la distorsion de la recherche du beau qui engendre un monstre.

13 De ce point de vue, Fils devient le moyen pour aborder la problématique du rapport entre l'original et la copie, thème particulièrement intéressant dans ce contexte. On trouve cet élément à la fin de la première partie du roman à propos de l'opération du rein subie par l'enfant. Après avoir implanté un troisième rein en croyant que le Fils en avait seulement un, Dolly est à nouveau victime d'une des crises de sa "maladie des possibilités infinies » et elle n'est plus capable de savoir avec certitude si l'enfant a trois ou deux reins. De plus: «[...] moi, dans mon imbécillité, je ne savais plus distinguer entre le rein original et celui que je lui avais implanté $»^{9}$. Le thème est repris dans la conclusion de la première partie du roman : 
מחוסר ברירה הותקנו הכבישים והמסילות החדשות לגובה, ושקשוק הרכבות שגבר

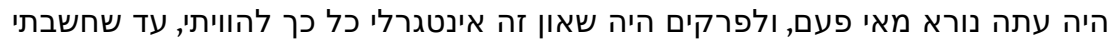

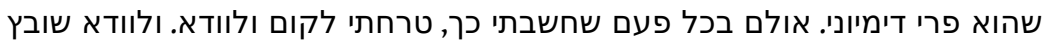

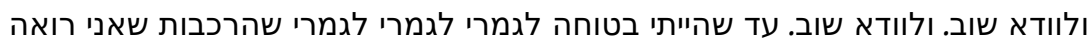

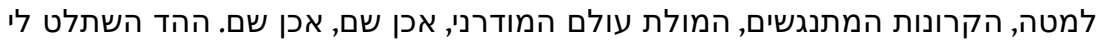

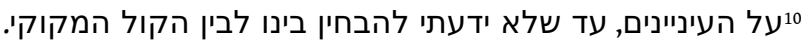

Dolly avoue qu'elle ne sait plus distinguer entre l'écho et le "vrai son », c'est-à-dire "ha-qol ha-meqori», la voix «originale». Cet adjectif nous renvoie au passage précédent: de la même façon que le bruit extérieur est tellement intégré à l'être de Dolly qu'elle en arrive à le considérer comme étant le fruit de son imagination, le rein qu'elle a implanté dans le Fils est devenu partie de son corps au point qu'elle n'est plus capable de le distinguer du corps « original » de l'enfant. Dans le cas de l'artiste et de sa création, s'instaure un écho qui est difficilement séparable du son orignal, qui appartient dans le même temps à la nature du créateur et à sa création, mais aussi qui arrive du monde extérieur.

Cet élément étrange affecte le créateur et en le même temps il est « un fruit de son imagination » en raison de son strict lien avec celui-ci. Il est intéressant de souligner le fait que cet élément est décrit comme une voix, comme «qol » et il est impossible de ne pas penser à la voix de Yona Wallach dans Ha-Tsippor (L'oiseau) poème où le conflit identitaire vécu par l'auteur est présenté comme conflit entre la voix originale de l'oiseau et le chant qui sort de la gorge de l'animal, mais qui ne lui appartient pas. Le thème de la définition du langage dans lequel on s'exprime et la difficulté de se l'approprier à cause de ses significations traditionnelles dans la culture et la société trouve ainsi écho dans le texte de Castel-Bloom. Cependant, à mon avis, l'auteure israélienne, sans oublier ces débats dans la littérature féminine, choisit de mettre en relief les différents aspects de ce rapport entre original et copie en proposant au lecteur d'autres éléments de réflexion.

16 Comme on vient de l'affirmer, le mythe de l'artiste se présente dans Dolly City « en coalescence ", pour citer encore Anne Geisler Szmulewicz, avec le mythe de Déméter et Perséphone, donc avec une perspective maternelle. Fils, dans sa double nature d'œuvre d'art, mais aussi de fils, incarne le drame de la création/procréation comme production/reproduction du même. Le conflit original-copie devient par rapport à l'identité de l'enfant «Fils » une allusion au thème du clone, au thème du Golem, donc d'une création comme clonage, comme production sans reproduction qui devient répétition. Sans banaliser, cet aspect est encore plus puissant si l'on considère la nature féminine de Dolly, cette poupée qui n'est plus mère, mais nourrice.

Dans cette opération de clonage artistique, ce qui est irrémédiablement perdu est le concept d'originalité, pour citer la pensée de Walter Benjamin telle qu'elle est présentée par Baudrillard:

Il faudrait reprendre ici ce que Walter Benjamin disait de l'œuvre d'art à l'ère de sa reproductibilité technique. Ce qui est perdu dans l'œuvre sériellement reproduite, c'est son aura, cette qualité singulière de l'ici et maintenait [...]. Ce qui est perdu, c'est l'original [...] ${ }^{11}$.

On revient donc à l'idée de simulation, de création comme production, d'impossibilité d'une création artistique autonome et originale et il est suffisant de citer le roman $\mathrm{Ha}$ Mina Liza $^{12}$ ou encore le protagoniste de la nouvelle Ha-soferet ke-zonat tsammeret ${ }^{13}$ pour démontrer combien le thème est fondamental dans la production littéraire d'Orly Castel-Bloom. La mécanisation du processus de création littéraire comme répétition et la négation d'une possibilité de création maternelle (ou peut-être l'appropriation de la 
maternité définie par Margaret Homans «circumvention of the maternal $»^{14}$ ) transforment la mère-artiste en un moyen pour créer/procréer, mais qui n'a plus aucun pouvoir sur ce processus.

Même l'obsession de Dolly pour le cancer peut assumer une nouvelle signification dans cette perspective, si l'on considère la définition que Baudrillard donne du processus de clonage: «L'individu n'est plus qu'une métastase cancéreuse de sa formule de base. Tous les individus issus par le clonage de l'individu $\mathrm{X}$ sont-ils autre chose qu'une métastase cancéreuse - prolifération d'une même cellule telle que l'on peut le voir dans le cancer? $»^{15}$. Fils n'as pas de nom, il est tout simplement «Fils», copie sans trop d'identité, produit, "golem», à savoir "corps sans forme", selon le dictionnaire hébreu d'Even-Shosan. Mais tous, hommes et objets, sont affectés par le cancer dans Dolly city :

ה־raison שלי שלח לי קונטרות : איך זה יכול להיות שלגלגלי המכוניות יש סרטן, אם

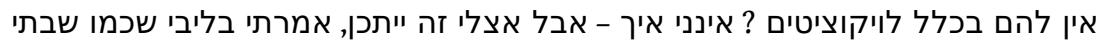

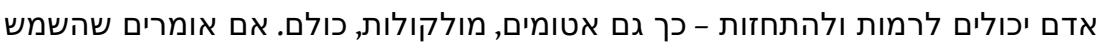

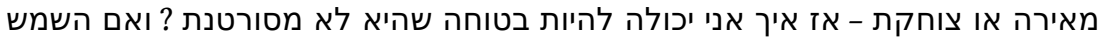

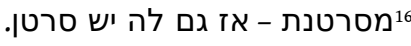

Création et procréation sont donc présentées dans le roman comme clonage, à savoir passage du même au même, répétition «à la lettre » du code. Peut-être même trop à la lettre, si l'on considère la force du phénomène de littéralisation dans ce roman, dont le passage ci-dessus est déjà un exemple très clair.

En effet, la réflexion au niveau de la créativité comme production devient, sur le plan du langage, une impossibilité de se détacher de la lettre. Le protagoniste nie la possibilité d'un langage métaphorique, les mots sont les choses, mais on se retrouve ainsi dans l'hyperréel de Baudrillard, dans un univers de simulation et non pas de représentation. On est donc plongé dans un langage qui, lié d'une façon trop étroite à la réalité pour masquer l'absence de réalité profonde, finit par être sans rapport avec quelque réalité que ce soit. Il est «son propre simulacre pur ». Le langage reste ainsi dénudé parce que le lien entre signifiant et signifié est déformé de façon à empêcher toute sorte de dialogue, comme l'épisode des derniers mots que le père adresse à la fille : le choix de Dolly d'aller étudier la médecine à Katmandu est la conséquence de son incapacité à comprendre la valeur ironique des derniers mots de son père mourant. Le langage perd donc sa fonction de système pour avoir accès à la réalité ou la représenter, une réalité dans laquelle Dolly cherche le serrurier dans la rue des Serruriers. La répétition, dont on a déjà traité pour ce qui concerne la créativité de l'auteur et la procréation de la femme, devient pour le langage une répétition des mots vidés de leur signification, procédé techniquement parfait, mais qui ne donne pas les résultats attendus : «Les rares personnes opérées par moi étaient mortes sur la table d'opération. L'opération avait réussi, mais le patient était mort $»^{17}$. Dans cette désolation, la stérilité des images, de signification, d'enfants, le roman Dolly city et la ville Dolly city sont une parfaite incarnation du simulacre, sont des gigantesques statues, monuments : «Les trains de Dolly City galopent vers Dachau et retour. Il ne s'agit pas de ce Dachau-là, mais d'une simple pancarte sur laquelle il est écrit Dachau, une espèce de mémorial $»^{18}$. 


\section{Conclusion}

et il me semble réducteur de lier ce procédé à la seule composante féministe. La puissance de ce Pygmalion-femme, mais surtout mère, qui cependant veut/doit créer comme un homme ou comme un scientifique, ouvre des horizons bien plus larges du mécanisme parfois banal de réécriture du mythe du point de vue féminin/féministe. Le thème de la stérilité, qui a joué un rôle important dans la tradition littéraire israélienne en tant que moyen pour définir une incapacité à laisser en héritage un système de valeurs comme en témoigne l'œuvre de Yaaqov Shabtai, devient ici un élément central du mythe de création/procréation devenu répétition stérile, mais aussi volonté d'être stérile.

L'idée d'inventio croise ici l'idée de tradition, de conservation dans la révolte ou de révolte dans la tradition. Même si le roman ne veut pas donner trop d'espérances, cette répétition stérile, « ces opérations sisyphiennes " selon les mots de Dolly, offrent au moins la possibilité de prendre conscience du procédé. On part donc du mythe et on y retourne aussi, mais sans plus croire à la possibilité de créer de nouveaux niveaux de signification, ou peut-être en feignant de se contenter de la mise en évidence de ce procédé. 


\section{BIBLIOGRAPHIE}

BAUDRILLARD, Jean (1981), Simulacres et simulation, Galilée, Paris.

BEN-YOSEF GINOR, Zvia (2000), « Ha-distopiah ha-tsiyyonit 'al-pi Orly Castel-Bloom », in Bar-El, Itzhaq, Schwartz, Yigal, Hes, Tamar (2000), Sifrut we-hevra ba-tarbut ha-'ivrit he-hadasha, Keter, Jérusalem.

CARANDINA, Élisa (2006), « Manifestazioni mostruose del materno : il mostro di Frankenstein di Mary Shelley e quello di Dolly City di Orly Castel-Bloom », in Annali di Ca' Foscari, 45.

CASTEL-BLOOM, Orly (1992), Dolly City, Zmora Bitan, Tel-Aviv. (Traduction française de PinhasDelpuech, Rosie (1993), Dolly city, Actes Sud, Arles).

CASTEL-BLOOM, Orly (1993) « Ha-soferet ke-zonat tsammeret », in Sippurim bilti-retsuniyyim, Zmora Bitan, Tel Aviv.

CASTEL-BLOOM, Orly (1995), Ha-Mina Liza, Keter, Jérusalem.

GEISLER-SZMULEWICZ, Anne (1999), Le Mythe de Pygmalion au XIX ${ }^{e}$ siècle, Éditions Champion, Paris. GINSBURG, Ruth (1997), “The Jewish Mother Turned Monster: Representation of Motherhood by Hebrew Women Novelists 1881-1993”, in Women's Studies International Forum, 20.

HOMANS, Margaret (1986), Bearing the Word: Language and Female Experience in XIX Women's Writing, University of Chicago Press, Chicago London.

HUTCHEON, Linda (1989), The Politics of Postmodernism, Oxford University Press, Toronto and New York.

IDEL, Moshe (1990), Golem: Jewish Magical and Mystical Traditions on the Artificial Anthropoid, Suny Press, Albany, New York.

McHALE, Brian (1987a), Postmodernist Fiction, Routledge, London and New York.

McHALE, Brian (1987b), Constructing Postmodernism, Routledge, London and New York.

SKERAH, Hanna (2000), « Ha-qol ha-mitologi shel ha-zehut ha-nashit ba-yetsiroten shel Ruth Almog we-shel Orly Castel-Bloom », in Alei-Siah 44.

\section{NOTES}

1. Voir : Baudrillard (1981), p. 10.

2. Pour une introduction cf. McHale (1987a \& b), Hutcheon, (1989).

3. Voir : Castel-Bloom (1992).

4. Le rapport entre le mythe du maternel et Orly Castel-Bloom dans le roman Dolly City a été déjà abordé par Hanna Skerah (2000), 19-41. Au contraire Zvia Ben-Yosef Ginor soutient dans son article «Ha-distopiah ha-tsiyyonit 'al-pi Orly Castel-Bloom» (2000), pp. 350-362, qu'Orly CastelBloom détruit tous les mythes en démontrant leur nature illusoire.

5. Voir : Ginsburg (1997), pp. 631-638.

6. Voir : Carandina (2006), pp. 51-65.

7. Voir : Geisler-Szmulewicz (1999).

8. Castel-Bloom, Dolly city, traduction française, p. 126 ; original hébreu, p. 76.

9. Ibid., traduction française, p. 64 ; original hébreu, p. 37. 
10. Ibid., original hébreu p. 38 ; traduction française p. 67 : «Faute de mieux, ces nouvelles routes et voies ferrées ont été installées en hauteur, et maintenant, les secousses croissantes des trains font un tel vacarme, un tumulte si intégré à mon être qu'il m'arrive de le prendre pour un fruit de mon imagination. Mais chaque fois que je l'ai cru, je me suis donné la peine de me lever pour le vérifier. Et encore vérifier. Et encore vérifier. Jusqu'au moment où j'étais tout à fait, tout à fait, tout à fait sûre que les wagons qui s'entrechoquent, la confusion du monde moderne est bien là, bien là, bien là. L'écho s'emparait alors de choses et je ne savais plus distinguer entre lui et le vrai son ».

11. Id., Simulacres et simulation, p. 149.

12. Voir : Castel-Bloom (1995).

13. Voir : Castel-Bloom (1993),pp. 109-114.

14. Voir : Homans (1986)

15. Id., Simulacres et simulation, p. 151.

16. Castel-Bloom, Dolly city, original hébreu, p. 50 ; traduction française, p. 84 : « Ma raison me faisait des tracasseries: si les pneus des voitures n'avaient pas de leucocytes, comment pouvaient-ils avoir le cancer? Je ne sais pas trop comment, mais pour moi c'était possible. Je me suis dit, de même que les êtres humains peuvent tricher et se déguiser, les atomes aussi le peuvent, et les molécules, et tout le reste. Si l'on peut dire que le soleil éclaire ou rit, alors comment puis-je être sûre qu'il n'est pas cancéreux ? Et s'il est cancérigène, alors c'est que lui aussi a le cancer ».

17. Ibid., traduction française, p. 37 ; original hébreu, p. 22.

18. Ibid., traduction française, p. 65 ; original hébreu, p. 37.

19. Voir : Idel (1990), p. 288.

\section{RÉSUMÉS}

Quelles sont les stratégies littéraires dans la littérature féminine israélienne ? Cet article traite de l'utilisation du mythe dans le roman Dolly City écrit par Orly Castel-Bloom. Plus précisément, il vise à approfondir la manière dont ce roman est construit sur l'idée de "répétition». Cet élément est facilement identifiable dans l'allusion au mythe de Pygmalion redéfini par Orly Castel-Bloom comme mythe de l'art, par la répétition au lieu de la représentation. Par ailleurs, l'utilisation maternelle du mythe de Pygmalion permet à Orly Castel-Bloom d'aborder le thème de la création et la procréation par le clonage, comme le personnage du Fils en forme de Golem pourrait le suggérer. Enfin, cette utilisation du mythe doit être comprise en comparaison avec la définition de « la création de mythes » révisionnistes » avancée par Alicia Ostriker.

This article deals with the use of myth in the novel Dolly City written by Orly Castel-Bloom. More specifically it aims to deepen how this novel is built on the idea of «repetition». This element is easily identifiable in the allusion to the Pygmalion myth redefined by Orly Castel-Bloom as myth of art as repetition instead of representation. Moreover the maternal use of Pygmalion myth allows Orly Castel-Bloom to approach the theme of creation and procreation as cloning, as the golem-shaped character of Son could suggest. Finally, this use of the myth is to be understood in comparison with the definition of «revisionist mythmaking» advanced by Alicia Ostriker. 


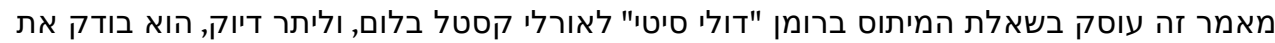

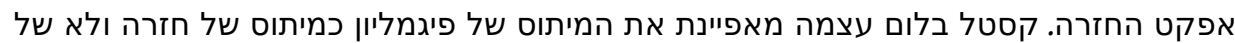

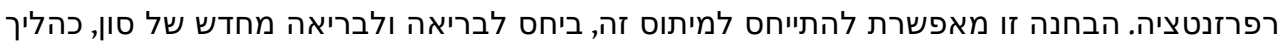
של שכפול. שימוש מעין זה במיתוס מצדיק עיון בתיאוריית "יצירת המיתוס זיתוס הרויזיזיזיוניסטיתטית" של אליסיה

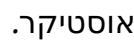

INDEX

מילות מפתח

שים בספרות, פמיניזם, החיפוש אחר הזהות, החברה הישראלית, ספרות, המאה:

העשרים, אורלי קסטל בלום, אמהות, אליסיה אוסטרייקר

Mots-clés : Castel-Bloom Orly (1960-), féminisme, quête identitaire, société israélienne, Ostriker Alicia (1937-), maternité

Index chronologique : vingtième siècle

Index géographique : Israël

Thèmes : littérature

Keywords : literature, twentieth century, Women in literature, feminism, quest for identity, Israeli society, Castel-Bloom Orly (1960-), Ostriker Alicia (1937-), maternity, Israel 